\title{
富勒烯和碳纳米管稳定性与形成机理的 图形理论定性研究"
}

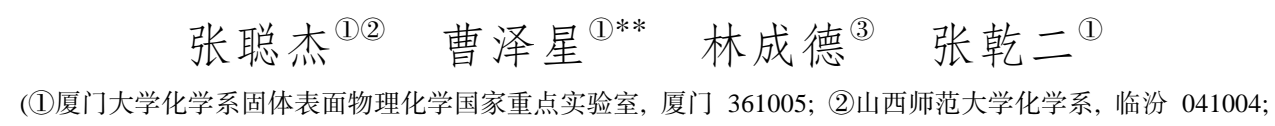
(3)厦门大学自动化系, 厦门 361005)

\begin{abstract}
摘要应用图形理论对不同种类碳簇体系的 Kekule 结构数进行了计算, 并在半经验 方法(AM1)和密度泛函理论(DFT)水平上, 讨论了不同种类碳簇的结构与稳定性. 基于 Kekule 结构计数, C-C $\sigma$ 键数, 富勒烯的表面曲率和能量, 对石墨碎片的卷曲行为以及 富勒烯的形成机理进行了讨论. 研究结果表明石墨碎片的卷曲, 一端闭合, 到完全封闭, 可以减少结构中的悬键; 随着新的 $\mathrm{C}-\mathrm{C} \sigma$ 键生成, Kekule 结构数将急剧地增加, 特别是 大的富勒烯和碳纳米管, 这种增加更为显著. 大量 Kekulé 结构间的共振使体系获得显 著的共振稳定化能, 稳定具有张力的富勒烯和碳纳米管, 并驱动平面碳簇结构向闭合 结构的转化. 对于 Kekule 结构数相近的碳笼, 表面曲率对曲面结构的稳定性有重要的 影响. 把 Kekule 结构计数和表面曲率结合起来, 可以合理地理解球形笼状富勒烯、闭 合纳米管和类 “洋葱”型结构等高碳族在热力学上的稳定性.
\end{abstract}

\section{关键词碳纳米管 富勒烯 Kekulé 结构数 稳定性}

富勒烯的形成机理主要有五元环途径 ${ }^{[1]}$, 萗环堆砌模型 ${ }^{[2,3]}$, 以及塌陷模型等 ${ }^{[4,5]}$. 利用两 端加“帽”的分子, Lagow等人制备了长达300个碳原子的长链炔烃, 通过对合成分子的研究, 他 们建议螺旋拉链形的碳炔 $-(\mathrm{C} \equiv \mathrm{C}-)_{m}$ 可能是形成富勒烯的先驱体. 然而, Brower等人的气相色 谱实验研究发现, 原子数 $n>20$ 的线性碳链分子的丰度非常之小.

石墨在高温下是不稳定的, 在 $2600 \mathrm{~K}$ 以上时将转变为多形态的碳炔(carbyne)形式, 形成炔 烃- $(\mathrm{C} \equiv \mathrm{C}-)_{m}$ 的可能机制如图 1 b-c 键断裂方式所示 ${ }^{[6]}$. 气相中, 石墨碎片通常存在很多悬键, 不可能保持平面结构, 将通过卷曲形成新的 C-C 键, 减少悬键, 使体系的能量达到最低 ${ }^{[7]}$. 此 外, 实验发现较大的偶数碳原子簇 $\mathrm{C}_{n}(40<n<80)$ 相对没有反应活性, 表明它们已经形成了没有 悬键的笼状结构 ${ }^{[8]}$.

1992年, 在电子束辐射条件下, Ugarte观察到了石墨结构的卷曲和闭合行为 ${ }^{[9]}$. 最初的产 物主要是管状和多面体的颗粒, 随着辐射的增加, 进一步转变为多层类“洋葱”型的结构, 层间 距离大约是 $0.334 \mathrm{~nm}$. 这些实验结果表明, 富勒烯在碳的气相中是通过消除悬键形成的.

对碳同素异形体的实验观察提出了一些基本的问题, 即, 富勒烯和碳纳米管等曲面(张力)

2003-06-23 收稿, 2003-08-25 收修改稿

* 国家自然科学基金(批准号: 20173042,90206038, 20233020 和 20021002)、教育部山西省青年基金(批准号: 20221012)和

福建省自然科学基金(A0010001)资助项目

**联系人, E-mail: zxcao@xmu.edu.cn 
结构高稳定性本质? 平面结构卷曲, 戴帽等结构变化的内在驱动力及其规律? 从结构化学的 观点看, 石墨, 富勒烯, 碳纳米管都是未饱和的 $\pi$ 电子离域体系, 它们的相对稳定性应该和 $\pi$ 电子的共轭性质有关. 根据共振论, Kekulé结构数较多的共轭体系应该更稳定. 如 $\mathrm{C}_{60}$ 巴基球包 含了大量的共振 Kekulé结构, 所以它是一种相当稳定的结构形式 ${ }^{[10]}$. 但是, 最近关于 $\mathrm{C}_{60}$ Kekulé结构计数与稳定性的关系引起了一些争议, 进而发展了一些新的共轭结构计数方法用 于稳定性的讨论. 例如Austin等人指出, 具有 $\mathrm{I}_{\mathrm{h}}$ 对称性的 $\mathrm{C}_{60}$ 不是Kekulé结构数最多的结构形 式 ${ }^{[11]}$, 但它具有最多的Fries Kekulé结构, 即所有的六边形包含三个双键, 而五边形没有双键 的Kekule结构. 对于二维网状碳结构, Zhu等人基于共轭圈计数, 对许多平面结构进行了研究.

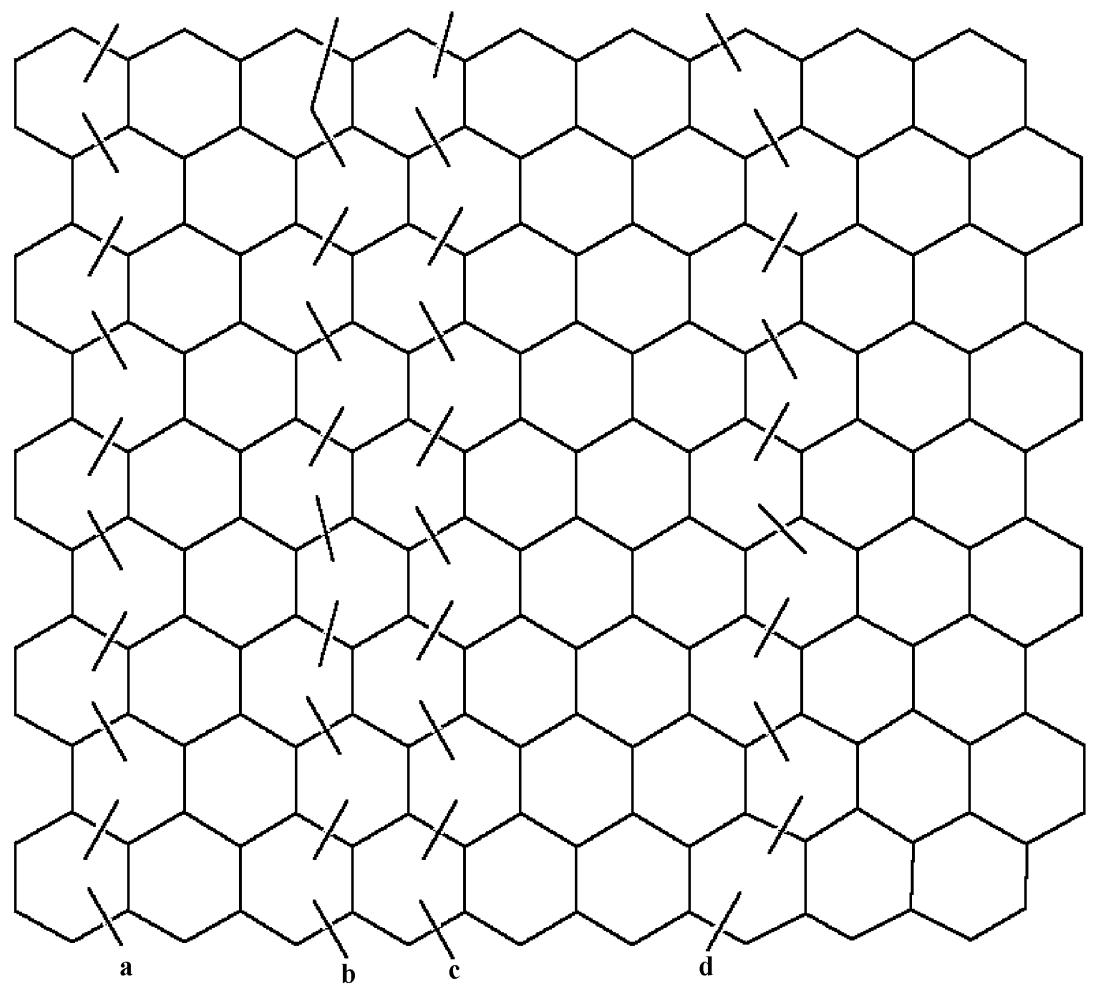

图1 石墨形成碎片的示意图

随着计算机技术的快速发展和量子化学计算方法的不断完善, 量子化学计算能力得到了 很大的提高, 可以处理较大的体系. 然而, 对于大的富勒烯和碳纳米管等复杂的碳簇结构, 进 行直接的理论计算研究仍然是不可能的. 为了理解碳簇不同结构形式的稳定性和构型变化性 质, 我们对 Kekulé 结构数与稳定性的关系进行了讨论, 结合曲面结构表面曲率的近似估算, 探讨了富勒烯和碳纳米管的稳定性与可能的形成机理.

\section{1 计算方法}

Kekulé 结构数可以通过枚举法和公式计算得到. 前者主要是基于图论的方法, 由于随着 体系的增大, 计算量急剧增加, 所以此方法不适合计算像富勒烯和碳纳米管这样大的体系. 除 了规则的苯环型碳氢分子外, 对一般的曲面碳簇结构, 很难获得一个计算 Kekulé 结构数的解 
析公式. 近来, 发展了一种基于 Kasteleyn 概念的有效算法, 可以计算富勒烯和单壁碳纳米管 (SWNT)等这样大的体系. 本文所有 Kekulé 结构数计算采用厦门大学自动化系林成德教授研 究小组开发的“Hex_Nanotube”程序，该程序包括了 Kasteleyn 算法、枚举法和公式法.

为了研究曲率对稳定性的影响, 我们定义笼状结构的曲率近似为其体积 $V$ 与表面积 $S$ 比 值, 即 $S / V$, 曲率的计算采用 AM1 方法优化得到的富勒烯结构. 为了比较不同碳簇结构的稳定 性, 在 6-31G*基组水平上, 应用 B3LYP 方法对 AM1 优化构型进行了单点能量计算, 所有的电 子结构计算采用 GAUSSIAN98 程序 ${ }^{[12]}$.

\section{2 结果与讨论}

在高温和激光气化条件下，石墨平面结构的断键方式可以有许多种. 如图 1 所示，除了先 前建议的 b-c 方式外 ${ }^{[6]}$, 还有 a-d 等其他可能的方式, 产生形式多样的石墨碎片或碳链. 这些 碎片作为其他结构的前驱体, 通过卷曲, 戴帽, 闭合等方式得到卷曲结构, 再逐渐转变为更稳 定的巨富勒烯 $\left(\mathrm{C}_{n}, n>100\right)$ 或碳纳米管等实验上观察到的碳同素异形体 ${ }^{[9]}$.

\subsection{Kekulé 结构数与稳定性}

先前关于碳氢化合物的理论研究表明, 其稳定性与 Kekulé 结构数存在着一定的关系. Kekule 结构数越多, 共轭分子所获得的共振稳定化能越大, 共轭体系越稳定. 因此, 可以假定 未饱和的纯碳簇和共轭的碳氢化合物一样, 趋向形成具有更多 Kekulé 结构数的曲面构型.

这里, 我们将以图 2 所示的 $\mathrm{C}_{20}$ 和 $\mathrm{C}_{24}$ 的几个平面结构为例, 来说明纯碳簇 Kekulé 结构数 与稳定性的关系. 表 1 给出了这些具有相同 C-C $\sigma$ 键数异构体的 Kekule 结构数和总能量. 从 表 1 不难发现, $C_{20}$ 和 $C_{24}$ 的稳定性顺序分别为: $C_{20}$ 1 $>C_{20}$ 2 和 $C_{24}$ 3 $>C_{24}{ }_{1}>C_{24}$ 2, 与它们的 Kekule 结构数完全一致. 说明 Kekulé 结构数越多, 碳簇越稳定. 这一结论对其他苯环型碳氢 化合物也是适用的, 因此, Kekulé 结构数可以衡量 $\pi$ 体系的稳定性.

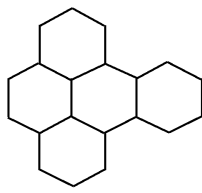

$\mathrm{C}_{20-1}$

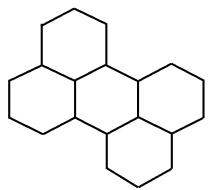

$\mathrm{C}_{20}{ }^{2}$

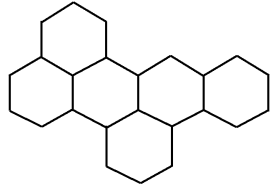

$\mathrm{C}_{24}{ }_{-1}$

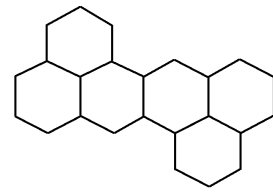

$\mathrm{C}_{24}{ }^{2}$

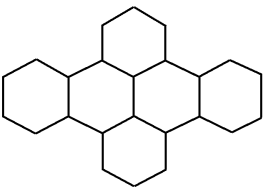

$\mathrm{C}_{24}{ }_{-} 3$

图 $2 \mathrm{C}_{20}$ 和 $\mathrm{C}_{24}$ 的几个结构

表 $1 \mathrm{C}_{20}$ 和 $\mathrm{C}_{24}$ 的 Kekulé 结构数 $(K), \mathrm{C}-\mathrm{C}$ 键数 $(N)$, 总能量 $(E /$ a.u. $)$ 和相对能 $\left(R E / \mathrm{kJ}^{\circ} \mathrm{mol}^{-1}\right)$

\begin{tabular}{lcrrcc}
\hline & 对称性 & $K$ & $N$ & $E$ & $R E$ \\
\hline $\mathrm{C}_{20 \_} 1$ & $\mathrm{C}_{2 \mathrm{v}}$ & 11 & 24 & -761.3166 & 0 \\
$\mathrm{C}_{20 \_}$ & $\mathrm{D}_{2 \mathrm{~h}}$ & 9 & 24 & -761.2783 & 100.8 \\
$\mathrm{C}_{24 \_}$ & $\mathrm{Cs}$ & 15 & 29 & -913.5673 & 130.2 \\
$\mathrm{C}_{24 \_} 2$ & 9 & 29 & -913.5621 & 142.8 \\
$\mathrm{C}_{24 \_}$ & $\mathrm{C}_{2 \mathrm{~h}}$ & 20 & 29 & -913.6168 & 0 \\
\hline
\end{tabular}

\section{2 碳笼的形成机制}

图 3 中列出了 $\mathrm{C}_{60}$ 的石墨碎片结构, 卷曲结构, 一端闭合和富勒烯 $\left(\mathrm{I}_{\mathrm{h}}\right)$ 结构, 分别用 $\mathbf{A}, \mathbf{B}, \mathbf{C}$ 
和 $\mathbf{D}$ 表示. 因为球型的 $\mathrm{C}_{60}$ 具有 $\mathrm{I}_{\mathrm{h}}$ 对称性, 其对应的平面结构应为 $\mathrm{C}_{2 \mathrm{~h}}$ 点群结构, 这将导致所 形成的巴基球结构中间层的六元环交替出现. $\mathrm{C}_{60}$ 的异构体 A-D 的 Kekule 结构数, C-C $\sigma$ 键数 和总能量都列在表 2 中, 从表 2 可以看出, 异构体 $\mathbf{A}, \mathbf{B}, \mathbf{C}$ 和 $\mathbf{D}$ 的 Kekulé 结构数从 2575(A)逐 渐增加到 $12500(D)$, 能量依次降低; $C_{70}, C_{80}$ 和 $C_{90}$ 也具有相同的递变规律. 为了探讨 Kekulé 结构数的变化与碳族大小的关系, 我们定义了平均 Kekulé 结构数, 即 $K / n$, 其中 $K$ 和 $n$ 分别表 示 Kekulé 结构总数和原子数. 图 4 给出了从石墨碎片到纳米管, 到一端闭合, 到完全闭合的纳 米管或富勒烯结构的 $K / n$ 与 $n$ 关系图. 从图 4 看出, $K / n$ 随着 $n$ 的增加而迅速增大. 尤其对于大 的闭合曲面结构, Kekule 结构数的增加十分显著, 大量 Kekule 结构间的共振可以使卷曲结构 获得显著的共振稳定化能. 因此, 对于大的碳簇, 闭合的曲面构型具有更高的稳定性, 为大碳 簇的优势构型, 这与实验观测结果一致 ${ }^{[5]}$.

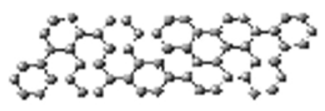

A

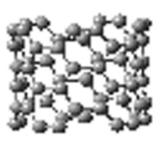

B

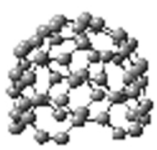

C

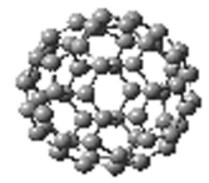

D

图 3 平面 $\mathrm{C}_{60}$ 可能的卷曲闭合过程

表 $2 \mathrm{C}_{60}, \mathrm{C}_{70}, \mathrm{C}_{80}$ 和 $\mathrm{C}_{90}$ 的 Kekulé 结构数 $(K), \mathrm{C}-\mathrm{C}$ 键数 $(N)$ 和相对能 $\left(R E / \mathrm{kJ}^{\circ} \mathrm{mol}^{-1}\right)$

\begin{tabular}{|c|c|c|c|c|c|c|c|c|c|c|c|c|}
\hline & \multicolumn{3}{|c|}{ 石墨碎片(A) } & \multicolumn{3}{|c|}{ 卷曲管(B) } & \multicolumn{3}{|c|}{ 戴一个“帽”(C) } & \multicolumn{3}{|c|}{ 富勒烯(D) } \\
\hline & $K$ & $N$ & $R E$ & $K$ & $N$ & $R E$ & $K$ & $N$ & $R E$ & $K$ & $N$ & $E$ \\
\hline $\mathrm{C}_{60}$ & 2575 & 77 & 0 & 3653 & 80 & -684.6 & 4768 & 85 & -2196.6 & 12500 & 90 & -3507.0 \\
\hline $\mathrm{C}_{70}$ & 1024 & 92 & 0 & 16401 & 95 & -907.2 & 28613 & 100 & -1835.4 & 52168 & 105 & -3641.4 \\
\hline $\mathrm{C}_{80}$ & 27907 & 106 & & 79594 & 110 & & 148596 & 115 & & 270153 & 120 & \\
\hline $\mathrm{C}_{90}$ & 3125 & 121 & & 468875 & 125 & & 722024 & 130 & & 1384341 & 135 & \\
\hline
\end{tabular}
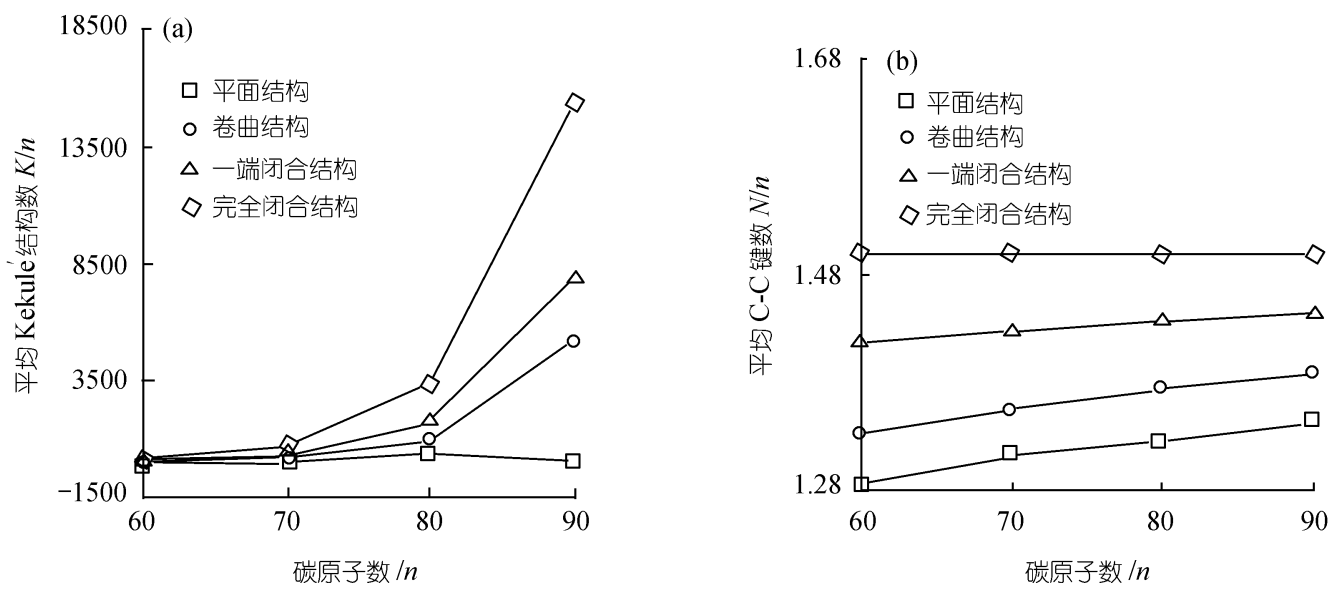

图 $4 \mathrm{C}_{60}, \mathrm{C}_{70}, \mathrm{C}_{80}$ 和 $\mathrm{C}_{90}$ 不同结构的平均 Kekulé 结构数和平均 C-C 键数

注意到平面结构在卷曲与闭合过程中, 将生成新的 C-C 键, 有利于体系能量的降低. 但卷 曲过程中, C-C 键的增加随碳簇的增大并不明显 (图 4 所示). 而且, 在闭合结构中, 原子的平 
均 C-C 键数达到极值 1.5 , 与碳簇大小无关. 进一步, 考虑到曲面结构的张力效应, 几个新的 C-C 键形成对整个体系的稳定化作用是有限的. 然而, 卷曲过程中端基碳原子的连通, 将导致 体系 Kekulé 结构数的急剧增加（图 4), 大量 Kekulé 结构间的共振所带来的稳定化能是显著的, 可以弥补石墨碎片卷曲时所产生的张力，稳定曲面结构.

\section{3 纳米管的 Kekulé 结构计数与卷曲方式}

图 5 给出了 $\mathrm{C}_{70}, \mathrm{C}_{90}$ 和 $\mathrm{C}_{110}$ 的石墨碎片和对应的 $(5,5)$ 型纳米管结构, 因为 $\mathrm{C}_{70}, \mathrm{C}_{90}$ 和 $\mathrm{C}_{110}$ 的富勒烯结构具有 $\mathrm{D}_{5 \mathrm{~h}}$ 点群, 所以它们的平面结构是 $\mathrm{D}_{2 \mathrm{~h}}$ 点群. $\mathrm{C}_{70}, \mathrm{C}_{90}$ 和 $\mathrm{C}_{110}$ 单壁碳管的 Kekulé 结构数列在表 3 中. 从表 3 结果可以看出, 对于不同的 $(m, n)$ 型纳米管, Kekulé 结构数差 别非常之大, 但 C-C 键数几乎一样; 且 $m$ 和 $n$ 的值相差越大, Kekulé 结构数越少, 只有 $(n, n)$ 型纳米管的 Kekulé 结构数最多. 因此, 按 $(n, n)$ 方式卷曲的碳纳米管更稳定, 容易形成, 这与实 验上观察到的结果一致 ${ }^{[13 \sim 15]}$.
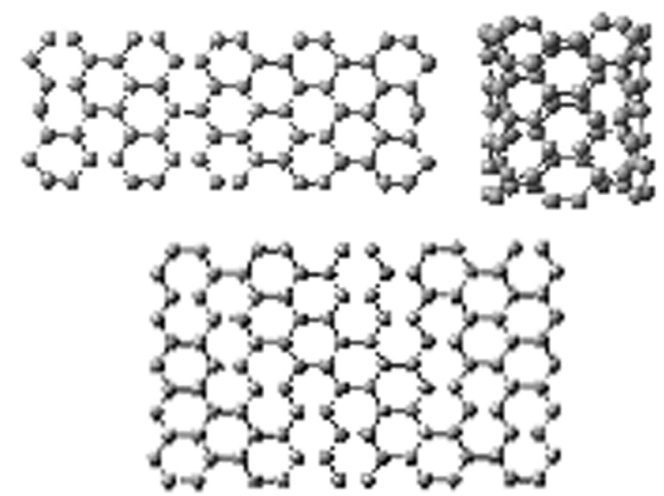

图 $5 \mathrm{C}_{70}, \mathrm{C}_{90}$ 和 $\mathrm{C}_{110}$ 平面和纳米管结构
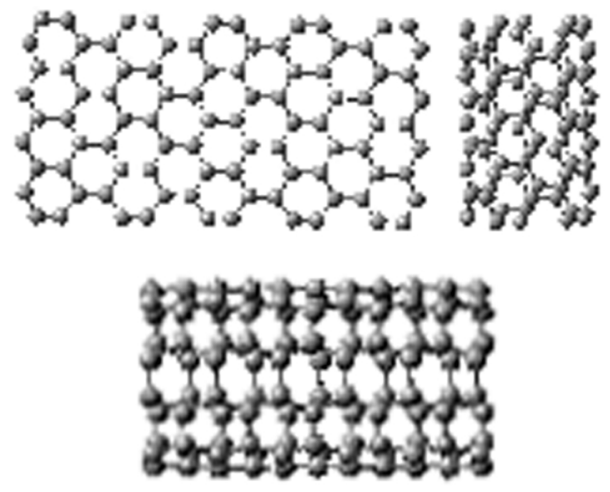

表 $3 \mathrm{C}_{70}, \mathrm{C}_{90}$ 和 $\mathrm{C}_{110}$ 不同卷曲模式的 Kekulé 结构数 $(K)$ 和 C-C 键数 $(N)$

\begin{tabular}{|c|c|c|c|c|c|c|}
\hline \multirow{2}{*}{ 卷曲方式 } & \multicolumn{2}{|c|}{$\mathrm{C}_{70}$} & \multicolumn{2}{|c|}{$\mathrm{C}_{90}$} & \multicolumn{2}{|c|}{$\mathrm{C}_{110}$} \\
\hline & $K$ & $N$ & $K$ & $N$ & $K$ & $N$ \\
\hline$(5,5)$ & 16401 & 95 & 468875 & 125 & 11691503 & 155 \\
\hline$(6,4)$ & 11619 & 94 & 347424 & 124 & 8225338 & 154 \\
\hline$(7,3)$ & & & 177880 & 123 & 4029057 & 153 \\
\hline$(8,2)$ & & & & & 1680249 & 152 \\
\hline
\end{tabular}

\section{4 曲率对稳定性的影响}

尽管每一个 Kekulé 结构之间存在一定的差异，对体系稳定化作用不一样，但当 Kekulé 结构计数相差巨大时, 这种差异对稳定性的影响可以忽略, Kekule 结构计数可以合理地预测 碳簇结构的稳定性. 然而, 如果不同碳簇构型 Kekulé 结构总数相差不大, 这时, 单个 Kekulé 结构上的差异, 曲面结构的弯曲程度等对稳定性的影响变得明显, 导致简单 Kekulé 结构计数 与稳定性的直接关系失效. 如 $\mathrm{C}_{60}$ 富勒烯不同笼状异构体, 其 Kekulé 结构总数相差不大, 且最 稳定 $\mathrm{I}_{\mathrm{h}}$ 结构的 Kekulé 结构总数并不是最高的 ${ }^{[11]}$.

为了讨论曲面结构曲率对稳定性的影响, 我们定义曲率为 $S / V$, 其中 $V$ 和 $S$ 分别表示笼状 结构的体积和表面积. $S / V$ 与曲面的半径 $r$ 相关联, 它可以衡量表面碳原子外向 $\mathrm{p}$ 轨道重叠积分 
的大小. $r$ 越大, 曲率越小, 重叠积分越大. 图 6 列出了 $\mathrm{C}_{60}$ 富勒烯的其他四个异构体(1 4). 这 四个异构体和 $\mathbf{D}$ 结构的 $S / V$ 值, Kekulé 结构数以及相对能都列在表 4 中, 从表 4 看出, Kekulé 结构数接近, $S / V$ 值与相对能量很好的一致, 即 $S / V$ 越小, 曲率越小, 能量越低.

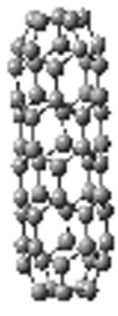

1

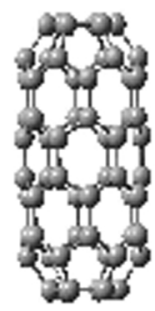

2

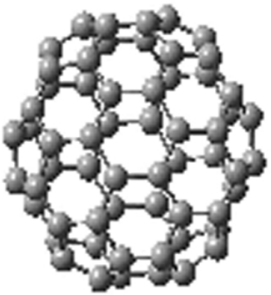

3

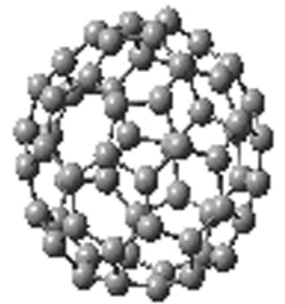

4

图 $6 \mathrm{C}_{60}$ 的其他四个异构体

对于具有相近 Kekulé 结构数的闭合笼状结构, Kekulé 结构数不能完全决定稳定性是个普 遍的现象. 事实上, $\pi$ 共轭体系的稳定性是由交换积分 $\beta$ (单个 $\pi$ 的强度)和 Kekulé 结构数共同 决定的. 当 Kekule 结构数没有明显差别时, 其稳定性就依赖于交换积分 $\beta$, 交换积分越大, 体 系越稳定. 交换积分与曲率有关, 曲率可以近似的用半径 $1 / r$ 来衡量. 当 $r \rightarrow \infty$ 时, 曲面就转变 为一个平面, 此时交换积分达到最大. $\mathrm{C}_{60} \mathrm{D}$ 结构的 $S / V$ 为 0.914 最小, 所以是最稳定的 $\mathrm{C}_{60}$ 构 型.

表 $4 \mathrm{C}_{60}$ 的五个异构体的 Kekulé 结构数 $(K)$, 体积 $\left(V / \mathrm{nm}^{3}\right)$, 表面积 $\left(S / \mathrm{nm}^{2}\right)$ 和相对能 $(R E /$ a.u. $)$

\begin{tabular}{ccccccc}
\hline 异构体 & 对称性 & $K$ & $V / 10^{-3}$ & $S / 10^{-2}$ & $(S / V) / 10$ & $R E$ \\
\hline $\mathbf{1}$ & $\mathrm{D}_{5 \mathrm{~d}}$ & 16501 & 118.283 & 152.136 & 1.287 & 0.899 \\
$\mathbf{2}$ & $\mathrm{D}_{6 \mathrm{~h}}$ & 12740 & 136.699 & 150.552 & 1.101 & 0.480 \\
$\mathbf{3}$ & $\mathrm{D}_{6 \mathrm{~h}}$ & 12688 & 157.148 & 150.792 & 0.960 & 0.321 \\
$\mathbf{4}$ & $\mathrm{D}_{5}$ & 9183 & 166.257 & 156.156 & 0.939 & 0.299 \\
$\mathbf{D}$ & $\mathrm{I}_{\mathrm{h}}$ & 12500 & 163.624 & 149.628 & 0.914 & 0.000 \\
\hline
\end{tabular}

\section{3 结论}

本文研究结果表明, 不同碳簇结构共轭体系的稳定性与 Kekulé 结构数, C-C 键数和表面曲 率密切相关; 石墨碎片的卷曲，闭合，到纳米管和富勒烯 “洋葱” 型结构的形成，可以消除端 基碳原子的悬键; 伴随少数 C-C 键的生成, 体系的 Kekule 结构数将急剧的增加, 增加的趋势 随体系的增大变得更为显著. 大量 Kekulé 结构数的增加所获得的共振稳定化能, 是富勒烯和 碳纳米管结构形成的驱动力. 对于 Kekulé 结构数相近的碳笼, 表面曲率是决定其结构稳定性 的主要因素之一. 把 Kekulé 结构计数和表面曲率结合起来, 可以合理地理解高碳簇在热力学 上倾向形成球形笼状富勒烯，闭合纳米管和类 “洋苾”型结构这一结构变化性质.

\section{参考文献}


2 Goeres A, Sedlmayr E. On the nucleation mechanism of effective fullerite condensation. Chem Phys Lett, 1991, 184(4): 310 317

3 Wakabayashi T, Achiba Y. A model for the $\mathrm{C}_{60}$ and $\mathrm{C}_{70}$ growth-mechanism. Chem Phys Lett, 1992, 190(5): 465 468

4 Heath J R in Fullerenes: Synthesis, Properties and Chemistry of Large Carbon Clusters. Hammond G S, Kuck V J, eds. Americian Chemical Society, Washington, DC, 1991. 1 23

5 Curl R F. Collapse and growth. Nature, 1993, 363(6424): 14 15

6 Whittaker A G. Carbon-new view of its high-temperature behavior. Science, 1978, 200(4343): 763 764

7 Robertson D H, Brenner D W, White C T. On the way to fullerenes: Molecular dynamics study of the curling of graphitic ribbons. J Phys Chem, 1992, 96(15): 6133 6135

8 Zhang Q L, O'Brien S C, Heath J R, et al. Reactivity of large carbon clusters: Spheroidal carbon shells and their possible relevance to the formation and morphology of soot. J Phys Chem, 1986, 90(4): 525 528

9 Ugarte D. Curling and closure of graphitic networks under electron beam irradiation. Nature, 1992, 359(6397): 707 709

10 Kroto H W, Heath J R, O'brien S C, et al. C60-buckminsterfullerene. Nature, 1985, 318(6042): 162 163

11 Austin S J, Flower P W, Hansen P, et al. Fullerene isomers of C $60_{60}$ Kekule counts versus stability. Chem Phys Lett, 1994, 228(4-5): 478 484

12 Frisch M J, et al Gaussian 98, Revision A7, Gaussian Inc Pittsburgh PA, 1998

13 Zhang S L. Optimal helicity of single-walled carbon nanotube. Phys Lett A, 2001, 285(3-4): 207 211

14 Thess A, Lee R, Nikolaev P, et al. Crystalline ropes of metallic carbon nanotubes. Science, 1996, 273(5274): 483 487

15 Terrones M, Grobert N, Olivares J, et al. Controlled production of aligned-nanotube bundles. Nature, 1997, 388(6637): 52 55 